\title{
GALACTIC CLUSTERS WITH ASSOCIATED CEPHEID VARIABLES. I. NGC 6087 AND S NORMAE
}

\author{
DAVID G. TURNER \\ Department of Astronomy, Saint Mary's University, Halifax, Nova Scotia B3H 3C3, Canada \\ Received 25 February 1986; revised 2 April 1986
}

\begin{abstract}
New photoelectric $U B V$ photometry is presented for stars in NGC 6087, the cluster containing the 9.75 Cepheid S Normae. The data are used with published photoelectric observations for cluster stars to redetermine the cluster reddening and distance by means of a careful application of the ZAMS fitting technique. The derived distance modulus, $V_{0}-M_{V}=9.78 \pm 0.03$ (internal error), \pm 0.13 (total estimated uncertainty), is shown to be in very good agreement with the cluster distance modulus obtained from Walraven photometry and from the analysis of Strömgren and $\mathrm{H} \beta$ photometry for cluster stars. However, the hydrogen-line data are found to produce more accurate and precise results if evolutionary corrections are ignored and replaced by due consideration of the cosmic scatter in $\mathrm{H} \beta$ distance moduli introduced by the effects of stellar rotation. The excellent agreement of the various distance estimates for NGC 6087 presented in this study results from the demonstrably identical zero points shared by the various luminosity calibrations used in the analysis. Internal consistency of this type has tended to be disregarded in some recent analyses of the Cepheid calibration clusters.
\end{abstract}

\section{INTRODUCTION}

A knowledge of the intrinsic colors and luminosities for galactic Population I Cepheids is of fundamental importance for the calibration of the Cepheid period-luminositycolor (PLC) relation, which in turn bears much of the weight in distance determinations for nearby spiral and irregular galaxies. Reliable estimates of reddening and distance can be obtained for Cepheids that are members of open clusters provided that in each case: (i) it can be shown that the Cepheid is indeed a cluster member, (ii) the quality and quantity of the photometric observations for cluster members are sufficient to determine the cluster distance unambiguously, and (iii) there are enough cluster members in the immediate vicinity of the Cepheid to determine its space reddening. Regrettably, the number of Cepheids that lie in well-studied clusters of this type is probably much smaller than is generally recognized. Many of the original broadband $U B V$ studies of the calibrating clusters tend to be rather incomplete due to undersampling of the faint cluster members used to determine the cluster distance, and sometimes they are affected by systematic errors in the photometry. The narrowband studies of these clusters are affected by other problems which will be discussed here.

This paper is the first in a series of new broadband photometric $U B V$ studies of clusters containing Cepheid variables. The ultimate aim of these studies is to resolve some of the current uncertainties in our knowledge of the distances and reddenings of these clusters. To this end, some effort has been made in the program to avoid problems of the type noted above by ensuring that: (i) observations are securely tied to the standard system, (ii) enough faint cluster members are observed to allow a reliable distance estimate to be made from zero-age main-sequence (ZAMS) fitting, (iii) several independent observations are available for each cluster member, and (iv) a concentration is made on stars lying in close proximity to the Cepheid. The present study is a new investigation of NGC 6087, the cluster containing the 9d75 Cepheid S Normae. Future papers in this series will examine a number of other clusters associated with Cepheids, including NGC 129, NGC 7790, Berkeley 58, NGC 5662, NGC 4349 , NGC 1647 and $\mathrm{C} 2128+488$, the cluster containing V1726 Cygni.
Previous broadband $U B V$ photoelectric studies of NGC 6087 have been published by lrwin (1958), Fernie (1961), and Breger (1966). However, none of these studies strictly satisfies all of the criteria for linear transformation to the $U B V$ system specified by Johnson (1963). This consideration is particularly important since it raises the question of how close the observed colors (most notably $U-B$ ) for cluster stars are to their true colors. Systematic errors in the photometry can contribute towards erroneous estimates for the reddening and distance of the cluster as obtained from standard photometric analyses, and might explain why Schmidt (1980) has found a distance to NGC 6087 using Strömgren and $\mathrm{H} \beta$ photometry for bright cluster stars that is $18 \%$ smaller than that inferred from ZAMS fitting of the $U B V$ data. This discrepancy amounts to a difference of 0.4 mag in distance modulus.

The new photoelectric $U B V$ study of NGC 6087 described here was initiated mainly to investigate this situation in more detail. This paper presents the new observational data from this study and also combines all useful data for cluster stars that have been published separately by various authors in order to determine the cluster reddening law, derive the reddening and distance of the cluster, and investigate in some detail the question of cluster membership for many individual stars. A much earlier investigation of this type by Landolt (1964) did not have nearly as much published data to draw upon.

\section{OBSERVATIONS}

The objects observed in this study comprise a sample of 31 stars in NGC 6087 lying in close proximity to S Nor. They were observed with the University of Toronto's $0.6 \mathrm{~m}$ telescope at Cerro Las Campanas, Chile, on three nights in 1981 May using a single-channel pulse-counting photometer equipped with a dry-ice-cooled 1P21 photomultiplier and a standard $U B V$ filter set. The precepts for $U B V$ photometry listed by Johnson (1963) were closely matched by the photometric system used for these observations, and as expected the transformation of instrumental colors and magnitudes to the Johnson system was quite linear with no indication of a need for second-order terms. Popper (1982) has noted that both the slopes and zero points of the color transformations 
give important information concerning the match of the instrumental system to the standard $U B V$ system. For the present observations the $B-V$ transformation had a slope of 0.97 and a zero-point correction of -1.13 , while the $U-B$ transformation had a slope of 1.02 and a zero-point correction of +0.72 . These values seem to indicate a relatively good match to the Johnson system for which zeropoint corrections of -1.0 and +1.1 , respectively, were adopted with the instrumental colors (cf. Popper 1982). There were also no obvious trends in the residuals in excess of an intrinsic scatter of about $\pm 0.01 \mathrm{mag}$ when the standard-star observations were plotted as functions of color. This again is indicative of a fairly good match to the Johnson system.

The data are presented in Table I, where the stars are identified according to the numbering systems of Landolt (1964), Fernie (1961), and Breger (1966). A few stars numbered by Landolt have accidentally been transposed in his tables of photometric data, so the star identifications used here refer to those specified by Fig. 2 of his paper. Cross identifications with the numbering system of Fernie (supplemented by Breger) are provided where possible to avoid confusion.

The $U B V$ data of Irwin (1958) for cluster stars have never been published. Therefore, a comparison of the present photometry with previously published observations is restricted to the data of Fernie (1961) and Breger (1966). A comparison with the photometry of Fernie yields the following differences (Turner - Fernie): $\Delta V=+0.008 \pm 0.007$,

TABLE I. New photoelectric data for NGC 6087.

\begin{tabular}{|c|c|c|c|c|c|}
\hline $\begin{array}{l}\text { Star Id } \\
\text { Landolt }\end{array}$ & $\begin{array}{l}\text { lentification } \\
\text { Fernie/Breger }\end{array}$ & V & $B-V$ & U-B & $n$ \\
\hline $\begin{array}{l}I-2-1 \\
I-2-2 \\
I-3-1 \\
I-3-2 \\
I-3-4\end{array}$ & $\begin{array}{l}64 \\
65 \\
35 \\
66 \\
68\end{array}$ & $\begin{array}{l}11.48 \\
12.08: \\
10.08 \\
12.11: \\
12.11:\end{array}$ & $\begin{array}{l}+0.14 \\
+0.23 \\
+0.39 \\
+0.25 \\
+0.24\end{array}$ & $\begin{array}{l}+0.08 \\
+0.22 \\
+0.20 \\
+0.23: \\
+0.25\end{array}$ & $\begin{array}{l}2 \\
2 \\
2 \\
2 \\
2\end{array}$ \\
\hline $\begin{array}{l}I-4-1 \\
I-4-2 \\
I-4-3 \\
I-4-7 \\
I-5-3\end{array}$ & $\begin{array}{r}7 \\
34 \\
92 \\
9 \\
.\end{array}$ & $\begin{array}{r}8.28 \\
11.10 \\
12.49 \\
9.44 \\
12.43\end{array}$ & $\begin{array}{l}+0.06 \\
+0.29 \\
+0.34: \\
+0.06 \\
+0.79\end{array}$ & $\begin{array}{l}-0.17 \\
+0.17 \\
+0.24 \\
-0.25 \\
+0.43\end{array}$ & $\begin{array}{l}2 \\
1 \\
2 \\
2 \\
1\end{array}$ \\
\hline $\begin{array}{l}\text { I-5-4 } \\
\text { II }-2-1 \\
\text { II }-2-2 \\
\text { II }-2-3 \\
\text { II }-2-5\end{array}$ & $\begin{array}{r}70 \\
10 \\
25 \\
128 \\
69\end{array}$ & $\begin{array}{r}13.10: \\
7.95 \\
10.01 \\
8.71 \\
12.20\end{array}$ & $\begin{array}{l}+0.44: \\
+0.07 \\
+0.11 \\
+0.05 \\
+0.27\end{array}$ & $\begin{array}{l}+0.24: \\
-0.34 \\
-0.26 \\
-0.40 \\
+0.22\end{array}$ & $\begin{array}{l}2 \\
4 \\
4 \\
2 \\
4\end{array}$ \\
\hline $\begin{array}{l}\text { II }-2-6^{a} \\
\text { II }-2-14 \\
\text { II }-2-15 \\
\text { II }-2-16 \\
\text { II }-3-1\end{array}$ & $\begin{array}{r}157 \\
11 \\
129 \\
70 \\
\ldots\end{array}$ & $\begin{array}{c}(12.78:) \\
9.39 \\
9.72 \\
11.97 \\
12.65\end{array}$ & $\begin{array}{l}(+0.49) \\
+0.04 \\
+0.19 \\
+1.53 \\
+0.29\end{array}$ & $\begin{array}{l}(+0.18) \\
-0.34 \\
+0.17 \\
+1.91: \\
+0.26\end{array}$ & $\begin{array}{l}4 \\
2 \\
2 \\
4 \\
2\end{array}$ \\
\hline $\begin{array}{l}\text { II }-3-2 \\
\text { II }-3-3 \\
\text { II }-6-1 \\
\text { II I }-2-5 N \\
\text { IV }-2-1\end{array}$ & $\begin{array}{r}\ldots \\
12 \\
39 \\
127\end{array}$ & $\begin{array}{r}12.56 \\
12.80 \\
7.46 \\
10.75 \\
10.19\end{array}$ & $\begin{array}{l}+0.29 \\
+1.32 \\
+1.17 \\
+0.84 \\
+0.09\end{array}$ & $\begin{array}{l}+0.24 \\
+1.36: \\
+0.97 \\
+0.46 \\
-0.29\end{array}$ & $\begin{array}{l}2 \\
2 \\
1 \\
2 \\
2\end{array}$ \\
\hline $\begin{array}{l}\text { IV }-2-1 S \\
\text { IV }-3-1 \\
\text { IV }-3-1 S W \\
\text { IV }-3-1 W a \\
\text { IV }-3-1 W b\end{array}$ & $\begin{array}{r}. \\
8 \\
130 \\
. . \\
. .\end{array}$ & $\begin{array}{r}11.50 \\
8.97 \\
9.15 \\
10.60: \\
11.32\end{array}$ & $\begin{array}{l}+0.34 \\
+0.04 \\
+0.04 \\
+0.40: \\
+0.15\end{array}$ & $\begin{array}{l}+0.22 \\
-0.37 \\
-0.37 \\
+0.23 \\
+0.04\end{array}$ & $\begin{array}{l}2 \\
2 \\
2 \\
2 \\
2\end{array}$ \\
\hline IV $-3-2$ & 36 & 10.38 & +0.07 & -0.23 & 2 \\
\hline
\end{tabular}

a Close companion to $S$ Normae. Data contaminated by stray light from $\mathrm{S}$ Normae. Not included in combined data of Table II.
$\Delta(B-V)=-0.030 \pm 0.004$ and $\Delta(U-B)=+0.067$ \pm 0.059 for five stars with $V<10$ (only three of these have $\bar{U}-B$ data $) ; \Delta V=+0.118 \pm 0.007$ and $\Delta(B-V)$ $=-0.055 \pm 0.008$ for six stars with $V>10$. The telescope and photometric equipment used by Fernie do not satisfy the criteria for linear transformation to the $U B V$ system specified by Johnson (1963), and it is probably not surprising that Fernie's observations exhibit systematic differences in magnitude and color with respect to the Table I data. These differences seem to be both magnitude and color dependent, as was found previously by Landolt (1964) from a comparison with Irwin's unpublished data, and by Breger (1966).

A comparison with the photometry of Breger yields the following differences (Turner - Breger): $\Delta V=-0.005$ $\pm 0.007, \Delta(B-V)=+0.005 \pm 0.003$ and $\Delta(U-B)$ $\equiv+0.055( \pm 0.006)+0.077(\bar{U}-B)_{\text {Breger }}$ for 19 stars. The linear color term in $U-B$ is a typical problem encountered with the photometric system used by Breger. However, it seems clear that his photometry agrees well with the Table I data once this is taken into account. The standard errors for the magnitude and color differences are no larger than expected for multiple photoelectric observations of the same stars, thus justifying a combination of the two data sets to obtain a more complete sample of photoelectric $U B V$ data. A compendium of the combined photometry for NGC 6087 stars is presented in Table II. A few stars observed only by Fernie are listed here because they are important to the analysis; their $B V$ data have been transformed to Irwin's system using Landolt's (1964) relations. The spectral classifications for cluster stars in this table are from Feast (1957), while proper-motion membership probabilities are taken from King (1982). The final column of Table II lists conclusions regarding the cluster membership of each star based upon the radial velocity (Feast 1957), proper motion, and photometric data.

\section{ANALYSIS}

The steps involved in a detailed analysis of $U B V$ data for open cluster stars (Turner 1981) include: (i) confirmation that the data are on the standard system, (ii) determination of the interstellar-reddening relation appropriate for the cluster, and (iii) corrections for interstellar reddening of individual stars prior to a ZAMS fit. It can be assumed from the discussion in Sec. II that the data of Table II are closely tied to the $U B V$ system. Full confirmation of this conclusion would require a number of independent sets of classical $U B V$ photometry of cluster stars for comparison, but these do not exist. The analysis of the cluster reddening relation also provides information about possible systematic errors in the photometry. In this case, the results appear to confirm that the data are closely tied to the standard system.

The reddening relation that applies to NGC 6087 was determined by three independent methods, namely: (i) from color excesses calculated for early-type cluster stars in Table II with reasonably certain spectral types, (ii) from color excesses calculated for early-type stars lying within $3^{\circ}$ of NGC 6087 for which both $U B V$ data (Klare and Neckel 1977; Schild et al. 1983) and MK spectral classifications (Garrison et al. 1977) are available, and (iii) using the observed colors of reddened dwarf stars from the tangent portion of the intrinsic color-color relation near spectral type A2. Method (iii) is a useful technique for clusters that contain early A type dwarfs, but strictly provides only a lower limit for the slope of the reddening relation. Its primary use- 
TABLE II. Compendium of observational data for NGC 6087.

\begin{tabular}{|c|c|c|c|c|c|c|c|c|c|c|c|c|}
\hline Star ${ }^{a}$ & V & $B-V$ & $U-B$ & $\mathrm{n}$ & MK & Sp. Type & $(B-V)_{0}$ & $E_{B-V}$ & $v_{0}$ & $P_{\mu}^{b}$ & $\mathrm{P}_{\mathrm{V}}^{\mathrm{c}}$ & $\mathrm{P}_{\mathrm{T}}^{\mathrm{d}}$ \\
\hline I-2-1 & 11.46 & +0.15 & +0.08 & 6 & . & & -0.03 & 0.18 & 10.90 & 90 & .. & M \\
\hline I $-2-2$ & 12.08 & +0.22 & +0.21 & 5 & . & & +0.04 & 0.18 & 11.52 & 94 & $\ldots$ & M \\
\hline$I-3-1$ & 10.09 & +0.39 & +0.18 & 5 & $\mathrm{~A} 2$ & V: & . & . & . & 0 & $M$ & NM \\
\hline$I-3-2$ & 12.10 & +0.24 & +0.25 & 5 & $\ldots$ & & +0.06 & 0.18 & 11.54 & 96 & . & M \\
\hline I $-3-3$ & 12.56 & +0.37 & +0.24 & 1 & $\ldots$ & & +0.21 & 0.16 & 12.06 & $\ldots$ & $\ldots$ & M \\
\hline$I-3-4$ & $12.11:$ & +0.24 & +0.25 & 2 & $\ldots$ & & +0.06 & 0.18 & 11.55 & 2 & .. & M \\
\hline$I-4-1$ & 8.28 & +0.06 & -0.18 & 5 & B9 & III: & -0.09 & 0.15 & 7.82 & 88 & M? & M \\
\hline I $-4-2$ & 11.10 & +0.29 & +0.17 & 1 & $\ldots$ & & $\ldots$ & $\ldots$ & $\ldots$ & 96 & . & NM \\
\hline $\mathrm{I}-4-3$ & 12.49 & $+0.34:$ & +0.24 & 2 & $\ldots$ & & +0.19 & 0.15 & 12.03 & 96 & . & M \\
\hline$I-4-7$ & 9.44 & +0.06 & -0.25 & 3 & B9 & V & -0.11 & 0.17 & 8.91 & 93 & $M$ & M \\
\hline I $-5-3$ & 12.43 & +0.79 & +0.43 & 1 & $\ldots$ & & & & & $\cdots$ & . & NM \\
\hline$I-5-4$ & 13.10: & $+0.44:$ & $+0.24:$ & 2 & .. & & +0.26 & 0.18 & 12.54 & . & . & M \\
\hline I $-6-1$ & 10.29 & +0.01 & -0.33 & 1 & $\mathrm{~A} 0$ & III: (V?) & -0.13 & 0.14 & 9.86 & 93 & M & M \\
\hline$I-8-3$ & 7.67 & +1.78 & +2.39 & 4 & M1 & III & . & $\ldots$ & . & 0 & M & $\mathrm{NM}$ \\
\hline I-9-2 & 10.63 & +0.06 & -0.18 & 3 & $\ldots$ & & -0.09 & 0.15 & 10.17 & 96 & $\ldots$ & M \\
\hline I I - 2-1 & 7.95 & +0.07 & -0.34 & 7 & B5 & $V(e)$ & -0.15 & 0.22 & 7.27 & 90 & M & M \\
\hline II $-2-2$ & 10.03 & +0.09 & -0.25 & 9 & . & & -0.13 & 0.22 & 9.35 & 96 & . & M \\
\hline I I $-2-3$ & 8.72 & +0.03 & -0.40 & 13 & B5 & V & -0.16 & 0.19 & 8.13 & 89 & M & M \\
\hline I I $-2-5$ & 12.18 & +0.28 & +0.24 & 6 & $\ldots$ & & +0.13 & 0.15 & 11.72 & . & . & M \\
\hline I I $-2-6$ & 12.84 & +0.34 & +0.12 & 2 & . & & . & $\cdots$ & . & . & $\cdots$ & $\mathrm{NM}$ \\
\hline II $-2-14$ & 9.40 & +0.03 & -0.34 & 6 & B5 & v & -0.14 & 0.17 & 8.87 & 95 & M & M \\
\hline II $-2-15$ & 9.74 & +0.18 & +0.17 & 6 & A0 & v & . & . & . & 96 & M & NM \\
\hline II $-2-16$ & 11.96 & +1.50 & +1.90 & 5 & $\ldots$ & & .. & . & . & . & $\ldots$ & NM \\
\hline I I - 3-1 & 12.65 & +0.29 & +0.26 & 2 & . & & . & $\ldots$ & . & . & $\ldots$ & $\mathrm{NM}$ \\
\hline II $-3-2$ & 12.56 & +0.29 & +0.24 & 2 & . & & +0.14 & 0.15 & 12.10 & 90 & .. & M \\
\hline I I $-3-3$ & 12.80 & +1.32 & +1.36 & 2 & . & & . & . & . & $\ldots$ & . & $\mathrm{NM}$ \\
\hline I I - 4-1 & 10.31 & +1.40 & +1.79 & 3 & . & & . & $\ldots$ & . & 90 & . & NM \\
\hline II $-4-4$ & 12.77 & +0.24 & +0.21 & 3 & . & & . & . & . & . & . & NM \\
\hline II $-4-7$ & 12.28 & +1.40 & $\therefore$ & 2 & $\therefore$ & & . & $\cdots$ & . & $\therefore$ & 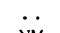 & NM \\
\hline I I $-6-1$ & 7.48 & +1.18 & +1.00 & 5 & G4 & II I & . & $\cdots$ & $\cdots$ & 87 & $\mathrm{NM}$ & $\mathrm{NM}$ \\
\hline II $-6-2$ & 8.71 & +1.74 & $+2.32:$ & 3 & M3 & II I : & & & & 0 & $M ?$ & $\mathrm{NM}$ \\
\hline II $-6-7$ & 11.90 & +0.17 & +0.13 & 1 & .. & & -0.01 & 0.18 & 11.34 & 95 & . & M \\
\hline I I-9-1 & 8.47 & +0.03 & -0.34 & 3 & $\ldots$ & & -0.13 & 0.16 & 7.97 & 88 & . & M \\
\hline I I $-10-2$ & 9.89 & +0.06 & 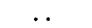 & 1 & B8 & V & -0.09 & 0.15 & 9.43 & 95 & M & M \\
\hline I I I - 2-1 & 11.39 & +0.13 & -0.02 & 1 & $\ldots$ & & -0.06 & 0.19 & 10.80 & 96 & $\ldots$ & M \\
\hline I I I $-2-5 \mathrm{~N}$ & 10.75 & +0.84 & +0.46 & 2 & $\ldots$ & & & $\ldots$ & .. & 0 & $\ldots$ & NM \\
\hline II I-3-1 & 9.70 & +0.09 & -0.23 & 3 & B8 & $V(e)$ & -0.11 & 0.20 & 9.08 & 95 & M & M \\
\hline III $-3-2$ & 11.41 & +0.18 & +0.12 & 3 & $\ldots$ & & -0.02 & 0.20 & 10.79 & 82 & . & M \\
\hline II I $-4-1$ & 9.29 & +0.03 & -0.38 & 3 & B5 & $\mathrm{V}$ & -0.15 & 0.18 & 8.73 & 95 & M & $M$ \\
\hline I I I $-5-1$ & 10.18 & +0.05 & -0.36 & 3 & B8 & V or III & -0.14 & 0.19 & 9.59 & 95 & M & M \\
\hline I I I $-8-1$ & 8.31 & +0.02 & -0.41 & 3 & B6 & V & -0.16 & 0.18 & 7.75 & 94 & M & M \\
\hline IV-2-1 & 10.19 & +0.09 & -0.29 & 6 & B8 & V & -0.14 & 0.23 & 9.48 & & M & M \\
\hline IV-2-IS & 11.50 & +0.34 & +0.22 & 2 & .. & & 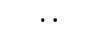 & 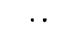 & .. & 92 & .. & NM? \\
\hline IV $-3-1$ & 8.99 & +0.03 & -0.38 & 5 & B6 & V & -0.15 & 0.18 & 8.43 & 96 & M & M \\
\hline IV-3-1SW & 9.18 & +0.05 & -0.36 & 5 & B7 & V & -0.15 & 0.20 & 8.56 & 77 & M & M \\
\hline IV $-3-1 \mathrm{Wa}$ & 10.60: & $+0.40:$ & +0.23 & 2 & .. & & . & . & $\ldots$ & 0 & . & NM \\
\hline IV $-3-1 W b$ & 11.32 & +0.15 & +0.04 & 2 & . & & -0.04 & 0.19 & 10.73 & 34 & . & M \\
\hline IV-3-2 & 10.38 & +0.07 & -0.24 & 5 & B8 & V & -0.12 & 0.19 & 9.79 & 95 & $M$ & M \\
\hline IV-3-7 & 11.02 & +0.13 & -0.03 & 3 & $\ldots$ & & -0.06 & 0.19 & 10.43 & 96 & $\ldots$ & M \\
\hline IV $-3-8$ & 11.45 & +0.22 & +0.18 & 3 & . & & +0.00 & 0.22 & 10.77 & 95 & . & M \\
\hline F 17 & 8.79 & +0.05 & .. & 1 & B8 & V & -0.10 & 0.15 & 8.32 & 0 & M & NM \\
\hline F 18 & 9.92 & +0.14 & . & 1 & $\mathrm{~B} 9$ & V & -0.03 & 0.17 & 9.39 & 91 & M & NM \\
\hline F 22 & 9.70 & +0.04 & $\ldots$ & 1 & $\ldots$ & & -0.15 & 0.19 & 9.11 & 0 & . & NM \\
\hline F 23 & 9.82 & +0.00 & . & 1 & . & & -0.16 & 0.16 & 9.32 & 95 & $\cdots$ & M \\
\hline $\mathrm{S}$ Normae & 6.41 & +0.95 & +0.64 & . & F8- & $-G 2 I b$ & +0.78 & 0.17 & 5.83 & 95 & M & M \\
\hline
\end{tabular}

a Identification from Fig. 2 of Landolt (1984).

b Proper motion membership probability (King 1982).

C Radial velocity membership (Feast 1957); $M=$ cluster member, $N M=$ non-member.

d Membership status from this study.

fulness is in providing a check on the consistency of the other estimates.

The results of this analysis are illustrated in Fig. 1. The implied slope $\mathrm{E}_{U-B} / \mathrm{E}_{B-V}$ of the reddening relation for NGC 6087 is unusually large for B- and A-type stars, but is confirmed by the good agreement of the three estimates. The reddening slopes $\mathrm{E}_{U-B} / \mathrm{E}_{B-V}$ resulting from each of the three methods were: (i) $0.83 \pm 0.07$ s.e., (ii) $0.85 \pm 0.03$ s.e., and (iii) $0.84 \pm 0.04$ s.e. (three stars). A value of $\mathrm{E}_{U-B} / \mathrm{E}_{B-V}=0.84$ was adopted in the analysis of NGC 6087 , and possible curvature in the relation was ignored since it is unimportant for small ranges of reddening like that found in NGC 6087.

The cluster color-color diagram is shown in Fig. 2, and clearly demonstrates the lack of any significant differential reddening across the region of NGC 6087. Likely cluster members and likely nonmembers are depicted by different symbols, where the assignment of cluster membership for individual stars was based upon their (i) radial velocity (Feast 1957), (ii) proper motion (King 1982), (iii) derived reddening from Fig. 2 or from dereddening their Strömgren colors, and (iv) implied distance modulus from their spec- 


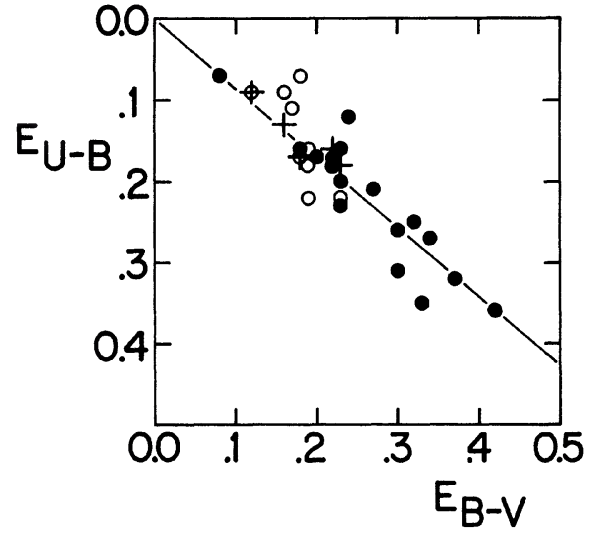

FIG. 1. Derived color excesses for stars in the field of NGC 6087 (dark circles) and in the cluster proper (light circles), and for likely cluster main-sequence stars lying near the tangent portion of the color-color relation (plus signs). The adopted reddening relation has slope $\mathrm{E}_{U-B} / \mathrm{E}_{B-V}=0.84$.

tral type, $\beta$ index or ZAMS fit. Membership status was usually obvious from these tests. Except for $S$ Nor, none of the late-type giants in the cluster region qualifies as a member of NGC 6087 on the basis of both radial velocity and proper motion. Different conclusions were reached by Landolt (1964) in his earlier study of NGC 6087.

A reddening-free color-magnitude diagram for NGC 6087 is given in Fig. 3, where extinction corrections were made using $R=A_{V} / \mathrm{E}_{B-V}=3.1$ as found for adjacent regions of the Milky Way (Turner 1976b). A fit to the ZAMS of Turner (1976a, 1979) implies that only seven of the observed stars are likely to be single, unevolved, main-sequence objects. The cluster distance modulus derived from these stars is $V_{0}-M_{V}=9.78 \pm 0.03$ s.e., which corresponds to a distance of $902 \pm 10 \mathrm{pc}$.

The Cepheid $\mathrm{S}$ Nor qualifies as a cluster member on the basis of its radial velocity (Stibbs 1955; Feast 1957) and proper motion. In addition, the implied age of the Cepheid

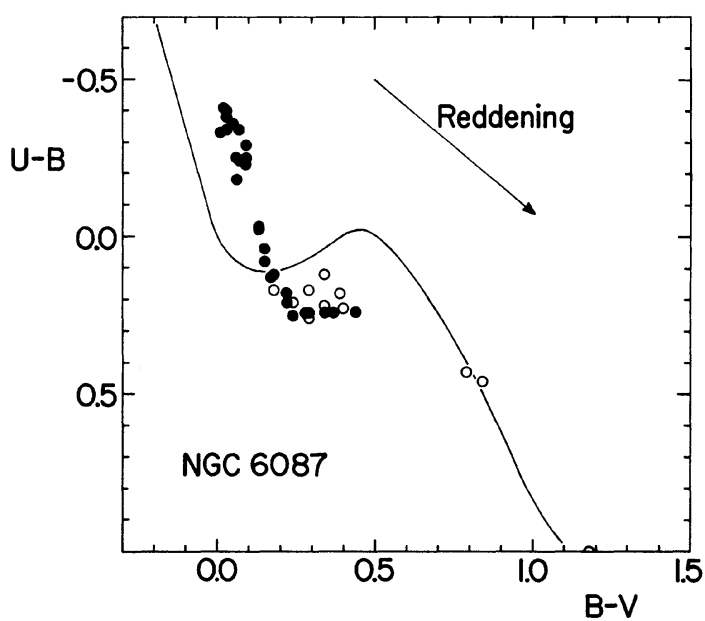

FIG. 2. Color-color diagram for NGC 6087 likely members (dark circles) and nonmembers (light circles). The continuous line is the intrinsic relation for main-sequence stars.

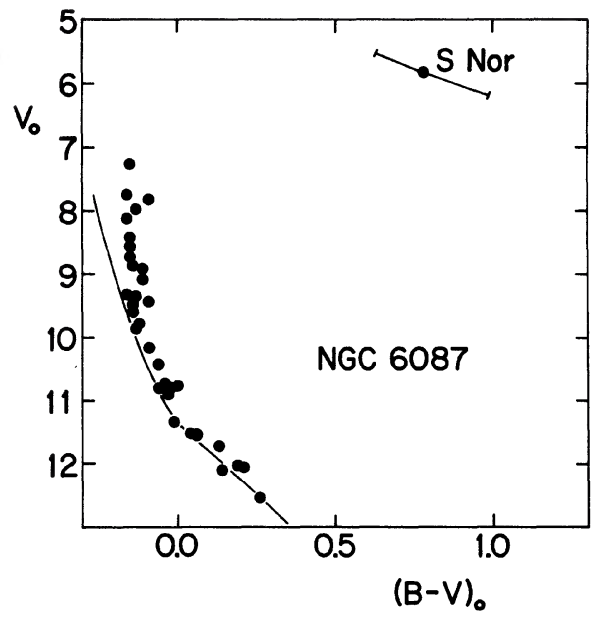

FIG. 3. Reddening-corrected color-magnitude diagram for NGC 6087 likely members. The extremes of variability for $\mathrm{S}$ Nor are indicated, while the continuous line represents the ZAMS for $V_{0}-M_{V}=9.78$.

from older stellar evolutionary models (Tammann 1970) is $4.1( \pm 0.8) \times 10^{7} \mathrm{yr}$, in good agreement with a cluster age of about $5( \pm 1) \times 10^{7} \mathrm{yr}$ corresponding to its main-sequence turnoff (cf. Maeder and Mermilliod 1981). The average field reddening of cluster members near $S$ Nor is $\mathrm{E}_{B-V}(\mathrm{~B} 0)=0.19 \pm 0.01$ s.e., or $\mathrm{E}_{B-V}=0.17 \pm 0.01$ for a star with the colors of $S$ Nor. A nearly identical reddening of $\mathrm{E}_{B-V}=0.18$ was derived for S Nor by Dean et al. (1978) using Cousins system $V R I$ photometry for the variable and an intrinsic color relation. Combined with the Cepheid's location at the heart of NGC 6087, these various pieces of evidence strongly suggest that $\mathbf{S}$ Nor is a cluster member, as has been assumed by previous workers. Its luminosity as a cluster member is $\left\langle M_{V}\right\rangle=-3.95 \pm 0.04$ s.e. (internal error), a value that is entirely appropriate for a classical Cepheid with a period of $9 ! 75$.

\section{THE ZERO-POINT QUESTION}

The cluster distance modulus of 9.78 is tied to a Hyades distance modulus of 3.15 (Turner 1979), but is based upon a fit to a ZAMS of solar metallicity by including a correction of +0.15 mag to the absolute magnitudes of Hyades stars to adjust them to Pleiades (solar) metallicity. The resulting ZAMS is very similar to older versions published by Johnson and Blaauw (cf. Stothers 1983) except for the earliest B- and O-type stars. More recent findings (Turner, unpublished; Pel 1985) indicate that the correction from Hyades to Pleiades metallicity is $0.21 \mathrm{mag}$ rather than $0.15 \mathrm{mag}$, which makes it possible to accommodate a Hyades distance modulus of 3.21 with no corresponding change in the distance derived for NGC 6087, assuming of course that a solar composition is appropriate for cluster stars. A recent summary by Hanson (1980) indicates that the Hyades distance modulus could be as large as 3.30 , but it is worth remarking that there still remain a number of sources of systematic error in the astrometric data quoted in this summary to consider a Hyades distance modulus of 3.2 a valid possibility. It will be pointed out in later papers in this series that the Cepheid clusters themselves provide some evidence in favor of a Hyades distance modulus of less than 3.3 (see also Stothers 1983; Schmidt 1984). 
Schmidt (1980) derived a distance modulus of $9.60 \pm 0.09$ for NGC 6087 from Strömgren and H $\beta$ photometry for 16 bright cluster stars ( 11 of which were adopted as cluster members) using Crawford's (1978) $M_{V}(\beta)$ calibration for B-type stars. Crawford's $M_{V}(\beta)$ scale is calibrated primarily using trigonometric parallaxes for $\mathrm{F}$ stars and not using stars in the Hyades cluster, yet it must have a zero point nearly identical to that quoted above according to the close similarity in distance moduli for the Pleiades cluster obtained by Crawford (1978) and Turner (1979). Balona and Shobbrook (1984) derived a comparable distance modulus of $9.64 \pm 0.09$ for NGC 6087 using Schmidt's data with their revised $M_{V}(\beta)$ calibration. In contrast, Pel (1985) obtained a distance modulus of $9.84 \pm 0.10$ for NGC 6087 from main-sequence fitting of Walraven photometry for the cluster. Pel's result is tied to a Pleiades distance modulus that is very similar to that found by Crawford (1978) and Turner (1979). The value of $9.78 \pm 0.03$ obtained here for the distance modulus of NGC 6087 lies closer to Pel's estimate than to those of Schmidt or Balona and Shobbrook, but the differences are slight and only half as large as the discrepancy of 0.4 mag inferred from Schmidt's study.

Older estimates for the distance modulus of NGC 6087 based upon ZAMS fitting are $9.8 \pm 0.1$ (Landolt 1964), 9.7 (Breger 1966), and 9.76 (Sandage and Tammann 1969). The last of these values was adopted for comparison by Schmidt (1980), but was corrected by $+0.26 \mathrm{mag}$ in order to adjust it to a Hyades distance modulus of about 3.3. As noted above, however, it is necessary to correct all previous cluster distance moduli by roughly $-0.2 \mathrm{mag}$ in order to place them on a ZAMS distance scale for stars of solar metallicity. Such a correction is necessary since the solar heavyelement abundance appears to be typical of stars in the Sun's galactic neighborhood (van den Bergh 1962, 1977). A correction for an upwards adjustment of the Hyades distance modulus is in addition to this metallicity correction, but could result in no net correction if the true distance modulus for the Hyades cluster is close to 3.2 (cf. Stothers 1983). At least 0.2 mag of the 0.4 mag discrepancy found by Schmidt (1980) in the distance moduli for NGC 6087 is therefore accounted for by his neglect of the metallicity correction. A similar omission was made by Fernie and McGonegal (1983) in their recent compilation of distance moduli for the Cepheid calibrating clusters.
The steep reddening relation for NGC 6087 obtained in Sec. III raises suspicions about previous analyses of the Strömgren system data for cluster stars, which were consistently reduced using "standard" reddening relations. Table III therefore presents a reanalysis of Schmidt's data using reddening relations for the Strömgren indices that are compatible with a reddening slope of $\mathrm{E}_{U-B} / \mathrm{E}_{B-V}=0.84$. The net difference in color excesses (Turner - Schmidt) amounts to only +0.004 mag in $\mathrm{E}_{b-y}$, which is much too small to be responsible for the residual discrepancy of 0.2 mag in the cluster distance modulus.

Column 6 of Table III lists the absolute magnitudes for each star, which are obtained from Crawford's (1978) $M_{V}(\beta)$ calibration. The resulting reddening-corrected distance moduli are plotted in Fig. 4(a) as a function of $10 \Delta \beta$, the parameter used in Crawford's evolutionary corrections for $M_{V}(\beta)$. Of the 16 cluster stars observed by Schmidt, two (stars 17 and 22) are nonmembers from the proper-motion data. The remaining 14 stars have proper-motion membership probabilities that average $93 \%$, and it appears that most are actual cluster members. Radial-velocity data confirm this for ten of the stars, while for one (star 7) the results are ambiguous (Feast 1957); three others (stars 20, 23, and 25) have no velocity measures available. Star 10 is a probable emission-line star according to Feast (the spectral type quoted by Schmidt is for another star), and it appears that its $\beta$ index is contaminated by weak emission; it also has a circumstellar component to its reddening which likewise argues that it is a Be star. This problem does not seem to exist for star 14, although it has a spectral classification similar to star 10

An initial analysis of the data for cluster stars would omit star 7, as permitted by the proper-motion and radial-velocity data, on the basis of its systematically large distance modulus compared to other cluster members. Data for the remaining stars (excluding star 10) result in a cluster distance modulus of $9.60 \pm 0.08$ s.e., which is identical to the value obtained by Schmidt except for a slightly smaller uncertainty (possibly accounted for by the different reddening analysis). Inclusion of the data for star 7 increases the mean distance modulus to $9.69 \pm 0.11$ s.e., but with a corresponding increase in the calculated uncertainty. The standard deviation for one star about the derived mean distance modulus is at best $\pm 0.29 \mathrm{mag}$ for the $\mathrm{H} \beta$ data, but is only \pm 0.08
TABLE III. Analysis of Schmidt's data for NGC 6087.

\begin{tabular}{rlccccccc}
\hline \hline Star & Landolt & $E_{b-y}$ & $10 \Delta B$ & $V_{0}$ & $M_{V}(\beta)^{a}$ & $V_{0}-M_{V}{ }^{a}$ & $M_{V}(\gamma)^{b}$ & $V_{0}-M_{V}{ }^{b}$ \\
\hline 1 & III-8-1 & 0.134 & 0.68 & 7.75 & -2.19 & 9.94 & -1.91 & 9.66 \\
7 & I-4-1 & 0.131 & 1.52 & 7.82 & -2.88 & 10.70 & -1.77 & 9.59 \\
8 & IV-3-1 & 0.129 & 0.46 & 8.43 & -1.20 & 9.63 & -1.16 & 9.59 \\
9 & I-4-7 & 0.117 & 0.43 & 8.91 & -0.50 & 9.41 & -0.46 & 9.37 \\
10 & II-2-1 & 0.169 & 1.44 & 7.27 & -5.26 & 12.53 & -3.83 & $11.10 \mathrm{e}$ \\
11 & II-2-14 & 0.138 & 0.39 & 8.87 & -0.92 & 9.79 & -0.95 & 9.82 \\
13 & III-4-1 & 0.132 & 0.24 & 8.73 & -0.64 & 9.37 & -0.80 & 9.53 \\
14 & III-3-1 & 0.138 & 0.40 & 9.08 & -0.46 & 9.54 & -0.44 & 9.52 \\
15 & III-5-1 & 0.129 & 0.11 & 9.59 & -0.24 & 9.83 & -0.53 & 10.12 \\
17 & $\cdots$ & 0.110 & 0.66 & 8.32 & -0.47 & 8.79 & -0.17 & 8.49 \\
18 &.- & 0.125 & 0.13 & 9.39 & +0.18 & 9.21 & -0.03 & 9.42 \\
19 & II-10-2 & 0.106 & 0.08 & 9.43 & +0.16 & 9.27 & -0.12 & 9.55 \\
20 & II-9-1 & 0.114 & 0.75 & 7.97 & -1.77 & 9.74 & -1.43 & 9.40 \\
22 & $\ldots$ & 0.142 & 0.93 & 9.11 & -4.46 & 13.57 & -3.61 & 12.72 \\
23 & $\cdots$ & 0.120 & 0.44 & 9.32 & -0.82 & 10.14 & -0.78 & 10.10 \\
25 & II-2-2 & 0.133 & 0.18 & 9.35 & +0.01 & 9.34 & -0.17 & 9.52 \\
\hline \hline
\end{tabular}

${ }^{a}$ Absolute magnitude from Crawford (1978) calibration.

b Absolute magnitude from Millward and Walker (1985) using $\beta$-index.

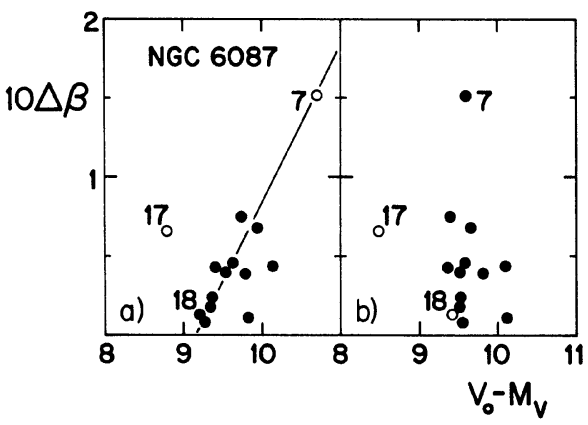

FIG. 4. Plot of Crawford's evolutionary correction term versus distance modulus for NGC 6087 stars observed by Schmidt, with adopted cluster members depicted by dark circles. Results are shown for a standard-type analysis (a) and for a new type of analysis described here (b). The line in (a) has unit slope. 
mag for the ZAMS fit. This last value corresponds to an uncertainty of about $\pm 0.015 \mathrm{mag}$ in $(B-V)_{0}$ for a cluster star, which is actually fairly typical of the internal scatter which arises in dereddened open cluster color-magnitude diagrams from using carefully analyzed, good quality, photoelectric data. The fourfold greater uncertainty in the distance modulus inherent to absolute magnitudes derived from Strömgren and $\mathrm{H} \beta$ photometry is larger than one would expect from photometric errors alone, and must result from large external errors in the luminosities. In fact, it is difficult to find any advantage to be gained from observations on the Strömgren and $\mathrm{H} \beta$ systems from comparisons such as this.

A disturbing feature of the data plotted in Fig. 4(a) is the apparent linear dependence of $V_{0}-M_{V}$ on $10 \Delta \beta$, the evolutionary correction for absolute magnitude. This dependence can be removed by ignoring the evolutionary correction term [cf. Fig. 4(b) ], but its presence raises the natural question of why evolutionary corrections are needed at all.

In Crawford's (1978) calibration the $10 \Delta \beta$ term was adopted as an adjustment to $M_{V}(\beta)$ in order to correct for a systematic trend evident in the distance moduli of the brightest stars in the Pleiades and $\alpha$ Persei clusters used as fundamental calibrators. A similar trend appears in the $M_{V}(\mathrm{H} \gamma)$ data of Millward and Walker (1985) for Pleiades and $\alpha$ Per cluster members, where it has again been attributed to evolutionary effects. However, the trends derived in these separate studies are not identical for the same clusters, and also seem to differ in character from the evolutionary trends obtained for these clusters by Crawford (1978) using luminosities derived from a $(U-B)_{0}$ versus $M_{V}$ (ZAMS) relation. A variety of effects have been considered at one time or another as important sources of external error in luminosities derived from hydrogen-line photometry (Schmidt 1984). Of these, the most important in our view seems likely to be stellar rotation and its direct effects on the continua and hydrogenline profiles of B type stars (Guthrie 1963; Collins and Harrington 1966; Hardorp and Strittmatter 1968). As discussed below, there are reasons for believing that stellar rotation is responsible for some of the systematic effects in hydrogenline luminosities that have been previously ascribed to evolution.

The implication from discussions by Crawford (1978), Schmidt (1984), and Millward and Walker (1985) concerning the effects of stellar rotation on hydrogen-line photometry for B type stars is that it should not introduce systematic errors in distance modulus, except perhaps for $V \sin i>250$ $\mathrm{km} \mathrm{s}^{-1}$, due to the compensating effect of random axial inclinations in a large sample of stars. Studies by Warren (1976) and Anthony-Twarog (1982) for members of Ori OB1 have substantiated this conclusion by demonstrating the lack of any systematic effects on the $\beta$ index for $V$ $\sin i<250 \mathrm{~km} \mathrm{~s}^{-1}$. However, it is noteworthy that very different conclusions were reached by Guthrie (1963) in an earlier study of the Orion association. The question of rotational influences on distance moduli for Orion stars was not specifically addressed in Warren's (1976) study, but did form the basis for the investigation by Anthony-Twarog (1982), albeit including Crawford's evolutionary corrections. It is conceivable that the distance spread in this association could mask any observable effect due to stellar rotation, particularly since the rotational velocities of Ori OB1 members are generally small. More importantly, the extreme youth of Orion stars contrasts noticeably with the ages of stars in clusters containing Cepheid variables. Effects that are similar to those observed in the Cepheid clusters should therefore be more clearly evident in clusters like the Pleiades and $\alpha$ Per clusters, which are closer in age to Cepheid variables.

A simple test of the importance of rotation to the determination of stellar luminosities from $\mathrm{H} \beta$ photometry was made here in the following manner. Strömgren and $\mathrm{H} \beta$ photometry for the Pleiades and $\alpha$ Per clusters, taken from Crawford and Perry (1976) and Crawford and Barnes (1974), respectively, were reanalyzed in the manner described previously for NGC 6087 stars using specific reddening relations compatible with the $U B V$ reddening relations derived for each cluster by Turner (1976b). Millward and Walker's $M_{V}(\mathrm{H} \gamma)$ calibration was next used to derive absolute magnitudes from the published $\beta$ indices using their relation between $\beta$ and $\mathrm{W}(\mathrm{H} \gamma)$. This calibration was adopted in preference to Crawford's calibration without evolutionary corrections since it was found to generate stellar distance moduli that exhibited smaller internal dispersion than applied to those obtained from the Crawford calibration when plotted as functions of the various parameters being tested. Its zero point appears to be identical to that applying to our adopted ZAMS. Published estimates of $V$ sin $i$ for cluster stars were obtained from the literature.

The effect of rotational distortion on a star's effective gravity for different angles of inclination is predicted to vary roughly as $V^{2} \sin ^{2} i$ (Collins and Harrington 1966; Hardorp and Strittmatter 1968; Warren 1976). The derived distance moduli for stars in the Pleiades and $\alpha$ Per clusters are therefore plotted as a function of this parameter in Fig. 5. A similar plot results from using Crawford's (1978) calibration without the evolutionary correction term.

There is a strong suggestion in the data of Fig. 5 that the stellar distance moduli depend linearly upon $V^{2} \sin ^{2} i$, at least for $V \sin i<250 \mathrm{~km} \mathrm{~s}^{-1}$. Above this value the rotational effects on $V_{0}-M_{V}$ appear to have "saturated" for $\alpha$ Per stars. Unfortunately, there is some scatter in the diagram which hinders this simple interpretation of the results. Part of this scatter may arise from the tendency for $\mathrm{H} \beta$ to become partially filled by emission for rapidly rotating stars (Craw-

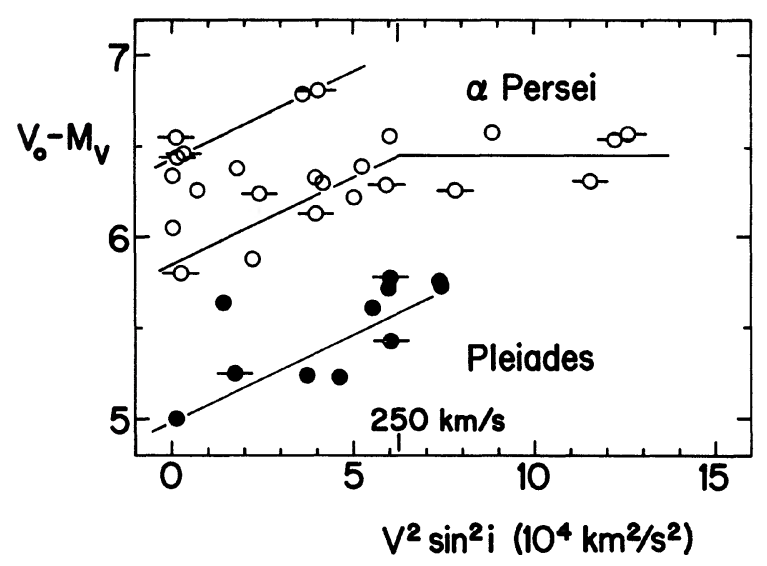

FIG. 5. Plot of distance modulus versus $V^{2} \sin ^{2} i$ for stars in the Pleiades (dark circles) and $\alpha$ Per (light circles) clusters. Bars denote recognized spectroscopic binaries. The distance moduli were obtained from $\mathrm{H} \beta$ photometry using the technique described in the text. Lines denote apparent trends in the data. 
ford 1978). This would result in systematically large distance moduli for cluster members which are extremely rapid rotators. In addition, the extensive area of sky occupied by the $\alpha$ Per cluster contains several background B stars that have not been eliminated from cluster membership by the radial-velocity studies (cf. Turner 1976b). They appear in Fig. 5 as a separate "cluster" with distance moduli $\sim 0.6$ mag larger than true $\alpha$ Per cluster members. Since recognized binaries in these clusters exhibit nearly the same dependence on $V^{2} \sin ^{2} i$ as do single stars, it is difficult to invoke stellar binarity as a possible explanation for the observed trends. Quite simply, it appears that stellar rotation does affect the absolute magnitudes obtained from hydrogen-line photometry, as argued previously by Collins and Harrington (1966) and Hardorp and Strittmatter (1968). The limiting accuracy in distance modulus if $V \sin i$ is not known for a star appears to be about $0.6 \mathrm{mag}$ from this effect. An inherent uncertainty of about $0.5 \mathrm{mag}$ was found by Hardorp and Strittmatter in their study.

The residual scatter superposed upon the stellar rotation trends is about $\pm 0.13 \mathrm{mag}$, which is probably governed by internal photometric errors. In fact, the total range in $V_{0}-M_{V}$ for stars in both the Pleiades and $\alpha$ Per clusters is $0.78 \mathrm{mag}$, which can be accounted for by the superposition of random photometric errors in the luminosities on the systematic spread in distance moduli resulting from cosmic variations in $V \sin i$ for cluster stars. The observed range in $V_{0}-M_{V}$ for NGC 6087 stars is very similar $[0.75 \mathrm{mag}$ from Fig. 4(b) ], which again must be due to the manner in which internal and external sources of error affect the hydrogenline luminosities.

Although it is essential to account for all possible sources of error in absolute magnitudes derived from hydrogen-line photometry, information on stellar rotational velocities is generally lacking for distant clusters such as NGC 6087. The approach adopted in Fig. 5 to study the Pleiades and $\alpha$ Per clusters is therefore of limited value for studying the Cepheid calibration clusters. It does illustrate, however, one of the serious problems limiting the accuracy of distance moduli derived for $\mathrm{B}$ stars from $\mathrm{H} \beta$ photometry. As indicated by the data of Figs. 4(a) and 5, it does not seem possible to account properly for the systematic effects resulting from a range of $V \sin i$ values for cluster stars by using an artificial evolutionary correction term.

Figure 4(b) presents the data of Table II, obtained from the Strömgren and $\mathrm{H} \beta$ photometry, analyzed using Millward and Walker's (1985) luminosity scale. Star 7 has been considered to be a cluster member here, while star 18 has been excluded as a probable nonmember. Although its proper motion, radial velocity, and distance modulus appear to be consistent with cluster membership, it falls well away from other stars when plotted in the cluster color-magnitude diagram (Fig. 3) and also lies in the outer part of the cluster. One of the high-membership-probability stars in Table III is permitted by the statistics to be a nonmember, and star 18 appears to be the most likely candidate.

Figure 6 presents the data of Fig. 5 in the same form used in Fig. 4, but with likely background stars in the $\alpha$ Per cluster field omitted. Similarly reduced data have also been included for stars in NGC 129, another Cepheid cluster. A full discussion of the data for this cluster is deferred to Paper II of this series.

The distribution of unreddened stellar distance moduli $V_{0}-M_{V}$ as a function of the "evolutionary" parameter

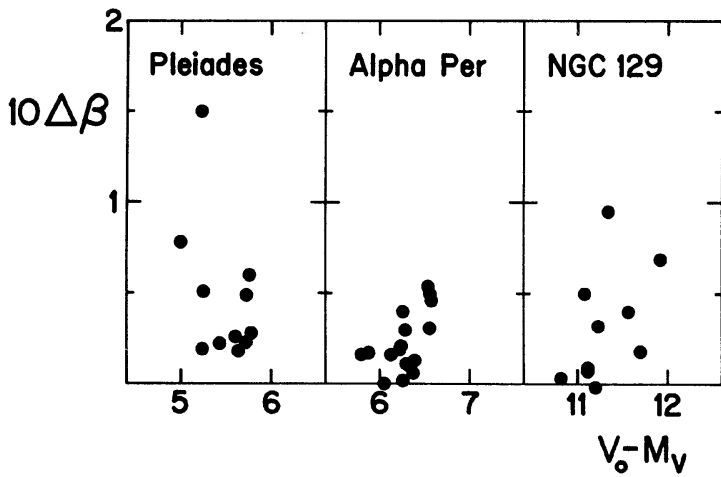

FIG. 6. Plot of Crawford's evolutionary correction term versus distance modulus for stars in the Pleiades, $\alpha$ Per, and NGC 129 clusters.

$10 \Delta \beta$ in Figs. 4 (b) and 6 is curiously different from one cluster to another, although some similarities are apparent. For example, NGC 6087 is similar to the Pleiades in the tendency towards decreasing distance modulus with increasing $\Delta \beta$. NGG 129 and $\alpha$ Per, on the other hand, exhibit trends in the opposite sense, possibly as a consequence of weak emission due to rapid rotation in a few of the stars. The principle of conservation of angular momentum dictates that stellar rotational velocities should decrease with increasing stellar radius as stars evolve away from the ZAMS. Since this would result in systematically small distance moduli according to Fig. 5, the trends evident for NGC 6087 and the Pleiades are in the correct sense for an evolutionary effect. Mean distance moduli for these clusters might be more appropriate if determined using stars exhibiting only minimal evolution or rotational displacement from the ZAMS, say for $10 \Delta \beta<0.5$.

The distance modulus of NGC 6087 obtained from the 12 stars in Fig. 4(b) considered as cluster members is $V_{0}-M_{V}=9.65 \pm 0.07$ s.e. If the three stars with $10 \Delta \beta>0.5$ are excluded from the mean, the result is $V_{0}-M_{V}=9.68 \pm 0.09$ s.e. This last value agrees within its uncertainty both with Pel's (1985) distance modulus of $9.84 \pm 0.10$ and with the cluster ZAMS-fitted distance modulus of $9.78 \pm 0.03$ presented earlier. Closer agreement of these values is clearly limited by the distribution of $V \sin i$ values for cluster stars. It is worth remarking that, according to the data of Fig. 5, a cosmic scatter of roughly $0.7 \mathrm{mag}$ to $0.8 \mathrm{mag}$ is expected in the distance moduli for cluster stars derived from $\mathrm{H} \beta$ photometry. The median distance modulus obtained from such data may therefore be the value which is closest to the true value, particularly if the distribution of $V \sin i$ values is skewed, as it appears to be for NGC 6087. The median distance modulus obtained from the $\mathrm{H} \beta$ photometry for NGC 6087 is $V_{0}-M_{V} \simeq 9.75$. This is virtually identical to the result obtained from ZAMS fitting, and removes almost all of the remaining 0.18 mag discrepancy in distance modulus for the cluster. There is therefore no evidence for a discrepancy in the cluster distance as obtained by the various methods, provided that the Strömgren and $\mathrm{H} \beta$ photometries are treated in the manner described here.

As a final step in the analysis, the Pleiades distance modulus has been recalculated from the data of Fig. 6. The mean distance modulus for all 11 cluster stars is $V_{0}-M_{V}=5.49 \pm 0.08$ s.e., but is $V_{0}-M_{V}$ $=5.57 \pm 0.08$ s.e. when restricted to the six stars (random- 
ly distributed in $V \sin i$ ) lying within the $10 \Delta \beta<0.5$ limit. This last value is identical to the Pleiades distance modulus obtained by Turner (1979) and that adopted by Pel (1985), and provides an independent argument for the excellent agreement in zero point of the various calibrations that have been used to derive the distance to NGC 6087. Since it is probably unrealistic to expect this zero point to be known to better than \pm 0.10 at the present time, a more reasonable value for the distance modulus of NGC 6087 is $V_{0}-M_{V}=9.78 \pm 0.13$ (total estimated uncertainty). This result is virtually identical to the older values derived by Landolt (1964), Breger (1966), and Sandage and Tammann (1969). However, it does represent a significant im- provement in general accuracy and internal consistency over what was possible with the techniques in use twenty years ago.

This investigation was supported by research funds awarded through the Natural Sciences and Engineering Research Council of Canada (NSERC), and was completed during the tenure of a NSERC University Research Fellowship. The assistance of Arlo Landolt in providing a correlation of published numbering schemes for NGC 6087 stars with the unpublished listing by John Irwin is most gratefully acknowledged.

\section{REFERENCES}

Anthony-Twarog, B. J. (1982). Astron. J. 87, 1213.

Balona, L. A., and Shobbrook, R. R. (1984). Mon. Not. R. Astron. Soc. 211, 375.

Breger, M. (1966). Publ. Astron. Soc. Pac. 78, 293.

Collins II, G. W., and Harrington, J. P. (1966). Astrophys. J. 146, 152.

Crawford, D. L. (1978). Astron. J. 83, 48.

Crawford, D. L., and Barnes, J. V. (1974). Astron. J. 79, 687.

Crawford, D. L., and Perry, C. L. (1976). Astron. J. 81, 419.

Dean, J. F., Warren, P. R., and Cousins, A. W. J. (1978). Mon. Not. R. Astron. Soc. 183, 569.

Feast, M. W. (1957). Mon. Not. R. Astron. Soc. 117, 193.

Fernie, J. D. (1961). Astrophys. J. 133, 64.

Fernie, J. D., and McGonegal, R. (1983). Astrophys. J. 275, 732.

Garrison, R. F., Hiltner, W. A., and Schild, R. E. (1977). Astrophys. J. Suppl. 35, 111.

Guthrie, B. N. G. (1963). Publ. R. Obs. Edinburgh 3, 81.

Hanson, R. B. (1980). In Star Clusters, IAU Symposium No. 85, edited by

J. E. Hesser (Reidel, Dordrecht), p. 71.

Hardorp, J., and Strittmatter, P. A. (1968). Astrophys. J. 153, 465.

Irwin, J. B. (1958). Astron. J. 63, 197.

Johnson, H. L. (1963). In Basic Astronomical Data, edited by K. A. Strand

(University of Chicago, Chicago), p. 204.

King, D. S. (1982). J. Proc. R. Soc. New South Wales 115, 1.

Klare, G., and Neckel, T. (1977). Astron. Astrophys. Suppl. 27, 215.
Landolt, A. U. (1964). Astrophys. J. Suppl. 8, 329.

Maeder, A., and Mermilliod, J. C. (1981). Astron. Astrophys. 93, 136. Millward, C. G., and Walker, G. A. H. (1985). Astrophys. J. Suppl. 57, 63. Pel, J. W. (1985). In Cepheids: Theory and Observations, IAU Colloquium No. 82, edited by B. F. Madore (Cambridge University, Cambridge), p. 1.

Popper, D. M. (1982). Publ. Astron. Soc. Pac. 94, 204.

Sandage, A., and Tammann, G. A. (1969). Astrophys. J. 157, 683.

Schild, R. E., Garrison, R. F., and Hiltner, W. A. (1983). Astrophys. J. Suppl. 51, 321.

Schmidt, E. G. (1980). Astron. J. 85, 158.

Schmidt, E. G. (1984). Astrophys. J. 285, 501.

Stibbs, D. W. N. (1955). Mon. Not. R. Astron. Soc. 115, 363.

Stothers, R. B. (1983). Astrophys. J. 274, 20.

Tammann, G. A. (1970). In The Spiral Structure of Our Galaxy, IAU Symposium No. 38, edited by W. Becker and G. Contopoulos (Reidel, Dordrecht), p. 236.

Turner, D. G. (1976a). Astron. J. 81, 97.

Turner, D. G. (1976b). Astron. J. 81, 1125.

Turner, D. G. (1979). Publ. Astron. Soc. Pac. 91, 642.

Turner, D. G. (1981). Astron. J. 86, 222.

van den Bergh, S. (1962). Astron. J. 67, 486.

van den Bergh, S. (1977). Astrophys. J. Lett. 215, L103.

Warren, W. H. (1976). Mon. Not. R. Astron. Soc. 174, 111. 